\title{
Recognizing Read-Once Functions from Depth-Three Formulas
}

\author{
Alexander Kozachinskiy \\ National Research University Higher School of Economics \\ akozachinskiy@hse.ru
}

\begin{abstract}
Consider the following decision problem: for a given monotone Boolean function $f$ decide, whether $f$ is read-once. For this problem, it is essential how the input function $f$ is represented. On a negative side we have the following results. Elbassioni, Makino and Rauf ([1]) proved that this problem is coNP-complete when $f$ is given by a depth- 4 read-2 monotone Boolean formula. Gurvich (2]) proved that this problem is coNP-complete even when the input is the following expression: $C \vee D_{n}$, where $D_{n}=x_{1} y_{1} \vee \ldots \vee x_{n} y_{n}$ and $C$ is a monotone CNF over the variables $x_{1}, y_{1}, \ldots, x_{n}, y_{n}$ (note that this expression is a monotone Boolean formula of depth 3; in [2] nothing is said about the readability of $C$, but the proof is valid even if $C$ is read-2 and thus the entire formula is read-3).

On a positive side, from 3 we know that there is a polynomial time algorithm to recognize read-once functions when the input is a monotone depth-2 formula (that is, a DNF or a CNF). There are even very fast algorithms for this problem ( [4]).

We show that we can test in polynomial-time whether a given expression $C \vee D$ computes a read-once function, provided that $C$ is a read-once monotone $\mathrm{CNF}$ and $D$ is a read-once monotone DNF and all the variables of $C$ occur also in $D$ (recall that due to Gurvich, the problem is coNP-complete when $C$ is read-2). We also observe that from the socalled Sausage Lemma of Boros et al. ([7]) it follows that the problem of recognizing read-once functions is coNP-complete when the input formula is depth-3 read-2. This improves the result of [1] in the depth and the result of [2] in the readability of the input formula. Moreover, we prove a new variant of Sausage Lemma, which allows us to derive that read-once recognition is coNP-complete even for depth-3 read-2 formulas of the form $\Psi \wedge D_{n}$, where $D_{n}$ is as above and $\Psi$ is a $\bigwedge-\bigvee-\wedge$ depth-3 read-once monotone Boolean formula.
\end{abstract}

Keywords: read-once functions, monotone Boolean functions, coNPcompleteness

\section{Introduction}

In this paper we study the following decision problem: decide, for a given monotone Boolean function $f$, whether $f$ is a read-once function (the latter means 
that the function can be computed by a monotone formula in which every variable occurs only once). Of course, to specify the problem, we need to specify the representation of $f$. For some representations this problem turns out to be tractable. For example, it is known (see [3]) that if $f$ is given by a monotone DNF (or, equivalently, CNF), then the corresponding problem can be solved in polynomial time. Golumbic, Mintz and Rotics (4) gave quite fast algorithm for this problem which works in time $O(n m)$, where $m$ is the length of the given $\mathrm{DNF}$ and $n$ is the number of variables. In [4] they also asked how hard is this problem when $f$ is represented in some other way, for example, when $f$ is given by an arbitrary Boolean formula.

First of all, let us note that if the input is a monotone Boolean formula, then the problem belongs to coNP. This follows from the following theorem by Gurvich:

Theorem 1 ([5]). A monotone Boolean function $f$ is read-once iff every minterm $S$ of $f$ and every maxterm $T$ of $f$ intersect in exactly one point.

Thus to show that $f$ is not read-once it is enough to demonstrate a minterm $S$ and a maxterm $T$ with $|S \cap T|>1$ (it is not hard to show that given a formula $S, T$, we can decide in polynomial time whether $S$ is a minterm and $T$ is a maxterm).

Soon after Golumbic, Mintz and Rotics raised their question, Elbassioni, Makino and Rauf (1]) proved that the read-once recognition problem is coNPcomplete when the input function $f$ is given by a depth- 4 read- 2 monotone Boolean formula. The same authors also proved that it is NP-hard to approximate the readability of the monotone Boolean function $f:\{0,1\}^{n} \rightarrow\{0,1\}$, given by a depth- 4 monotone Boolean formula, within a factor of $O(n)$.

Later, Gurvich ([2]) proved, that the problem of recognizing read-once functions is coNP-complete even when the input is the following expression: $C \vee D_{n}$, where $D_{n}=x_{1} y_{1} \vee \ldots \vee x_{n} y_{n}$ and $C$ is a monotone CNF over the variables $x_{1}, y_{1}, \ldots, x_{n}, y_{n}$. Note that the entire formula $C \vee D_{n}$ is a depth-3 formula. The paper [2] says nothing about the readability of $C$, but the proof is valid even if $C$ is read-2 1 (so that the whole expression is read-3).

Our main result shows that this problem becomes tractable, if $C$ is read-once even when $D_{n}$ is any monotone read-once DNF:

Theorem 2. There is a polynomial-time algorithm which decides, whether a given expression $C \vee D$ computes a read-once function, provided that $C$ is a monotone read-once CNF, $D$ is a monotone read-once DNF, and every variable of $C$ occurs also in $D$.

We don't know whether the last restriction can be removed. However, we find this theorem interesting due to its connection to the result of Gurvich.

We also observe that from the so-called Sausage Lemma of Boros et al. ([7]) it follows that the problem of recognizing read-once functions is coNP-complete

${ }^{1}$ This is due to the fact that the SAT-problem is NP-complete even for read-3 (nonmonotone) CNFs. 
when the input formula is depth-3 read-2 (note that [1] requires depth at least 4 and 22 requires readability at least 3 for the input formula). Moreover, we may consider only formulas which are conjunctions of two read-once formulas.

In more detail, Sausage Lemma states that it is coNP-complete problem to decide whether $\Psi \rightarrow D$ is a tautology, where $\Psi$ is a $\bigwedge-\bigvee-\bigwedge$ depth-3 read-once monotone Boolean formula and $D$ is a read-once monotone DNF. Further, it is easy to show (using Theorem 1) that $\Psi \rightarrow D$ is a tautology if and only if the following formula:

$$
\Psi \wedge\left(w_{1} w_{3} \vee w_{2} w_{4}\right) \wedge\left(D \vee w_{1} w_{2} \vee w_{3} w_{4}\right)
$$

computes a read-once function. Here $w_{1}, w_{2}, w_{3}, w_{4}$ are fresh variables.

Using a completely different reduction, we prove that Sausage Lemma is true even when $D$ is of the same form as in the Gurvich's hardness result from [2].

Theorem 3. The problem to decide whether $\Psi \rightarrow D_{n}$ is a tautology is coNPcomplete. Here $D_{n}=x_{1} y_{1} \vee \ldots \vee x_{n} y_{n}$ and $\Psi$ is a $\Lambda-\bigvee-\Lambda$ depth-3 read-once monotone Boolean formula over $x_{1}, y_{1}, \ldots, x_{n}, y_{n}$.

The same trick with fresh variables allows us to derive the following

Corollary 1. It is a coNP-complete problem to decide whether a given expression $\Psi \wedge D_{n}$ computes a read-once function. Here $D_{n}=x_{1} y_{1} \vee \ldots \vee x_{n} y_{n}$ and $\Psi$ is $a \bigwedge-\bigvee-\bigwedge$ depth-3 read-once monotone Boolean formula over $x_{1}, y_{1}, \ldots, x_{n}, y_{n}$.

Remark. Corollary 1 can be used to show that inapproximability result of Elbassioni, Makino and Raur is also true for depth-3 formulas. This is, however, can be done with the use of the result of Gurvich as well.

\section{Preliminaries}

A monotone Boolean formula (i.e., a $\wedge, \vee$-formula) $\Phi$ is called a read- $k$ formula if every variable occurs at most $k$ times in $\Phi$. A monotone Boolean function $f$ is called a read- $k$ function if there is a monotone read- $k$ formula, computing $f$. Readability of a Boolean function $f$ (formula $\Phi$ ) is the minimal $k$ such that function $f$ (formula $\Phi$ ) is read- $k$.

Assume that $f$ is a monotone Boolean function over the variables $x_{1}, \ldots, x_{n}$ and $S$ is a subset of $\left\{x_{1}, \ldots, x_{n}\right\}$. To simplify notation below let $f(S \rightarrow i)$ (here $i \in\{0,1\}$ ) denote the value of $f$ when all the variables from $S$ are set to $i$ and all the variables from $\left\{x_{1}, \ldots, x_{n}\right\} \backslash S$ are set to $1-i$.

A subset $S \subset\left\{x_{1}, \ldots, x_{n}\right\}$ is called a minterm of $f$ if $f(S \rightarrow 1)=1$ but for every proper subset $S^{\prime}$ of $S$ it holds that $f\left(S^{\prime} \rightarrow 1\right)=0$. Similarly, a subset $T \subset\left\{x_{1}, \ldots, x_{n}\right\}$ is called a maxterm of $f$ if $f(T \rightarrow 0)=0$ but for every proper subset $T^{\prime}$ of $T$ it holds that $f\left(T^{\prime} \rightarrow 0\right)=1$.

Obviously, every minterm of $f$ intersects every $f$ 's maxterm. 


\section{Proof of Theorem 2}

Our algorithm uses the following lemma:

Lemma 1. There exists a polynomial-time algorithm which for any given readonce monotone CNF $C$ and for any given read-once monotone DNF D decides, whether $C \rightarrow D$ is a tautology.

Proof. It is known (see, e.g., 6]) that there is a polynomial-time algorithm to decide, whether a given read-2 CNF is satisfiable. Apply this algorithm to $\neg(C \rightarrow$ $D)=C \wedge \neg D$ (the latter can be re-written as a read-2 CNF in polynomial time).

Let $\left\{x_{1}, \ldots, x_{n}\right\}$ be variables occurring in $D$. Let $C_{1}, \ldots, C_{m}$ denote the clauses of $C$. Since $C$ is read-once, we may identify $C_{1}, \ldots, C_{m}$ with $m$ disjoint subsets of $\left\{x_{1}, \ldots, x_{n}\right\}$. The same thing can be done for $D$. Let

$$
D=D_{1} \vee D_{2} \vee \ldots \vee D_{l}
$$

where $D_{1}, \ldots, D_{l} \subset\left\{x_{1}, \ldots, x_{n}\right\}$ are disjoint conjunctions. Note also that $D_{1} \cup$ $\ldots \cup D_{l}=\left\{x_{1}, \ldots, x_{n}\right\}$.

We provide first a description of minterms of $C \vee D$. Let $S$ be a subset of $\left\{x_{1}, \ldots, x_{n}\right\}$. We say that $S$ is a right set if for some $j \in\{1, \ldots, l\}$ we have $S=$ $D_{j}$. We say that $S$ is a left set if $S \subset C_{1} \cup \ldots \cup C_{m}$ and for every $i \in\{1, \ldots, m\}$ it holds that $\left|C_{i} \cap S\right|=1$. The following lemma is straightforward.

Lemma 2. A set $S$ is a minterm of $C \vee D$ if and only if $S$ is a left set that does not properly include any right set or $S$ is a right set that does not properly include any left set.

A minterm $S$ of $C \vee D$ is called a left minterm if $S$ is a left set. Similarly, we call $S$ a right minterm if $S$ is a right set.

Now we are ready to present the algorithm for Theorem 2 In the description of the algorithm we will state several auxiliary lemmas whose proofs are deferred to Appendix.

Algorithm. The algorithm works in four steps.

Step 1. Check, using Lemma 1, whether $C \rightarrow D$ is a tautology. If it is, then $C \vee D$ is equivalent to $D$ and hence $C \vee D$ computes a read-once function and the algorithm halts. Otherwise proceed to Step 2.

Step 2. Obviously every maxterm $T$ includes at least one clause of $C$. For every pair of distinct clauses $C_{u}, C_{v}$ check whether there is a maxterm $T$ of $C \vee D$ such that $C_{u}, C_{v} \subset T$. This can be done in polynomial time by the following

Lemma 3. Let $C_{u}, C_{v}$ be two distinct clauses of $C$. Then there exists a maxterm $T$ of $C \vee D$ such that $C_{u}, C_{v} \subset T$ if and only if for every $j \in\{1, \ldots, l\}$ it holds that $\left|\left(C_{u} \cup C_{v}\right) \cap D_{j}\right| \leq 1$. 
If there is such $T$, then $C \vee D$ is not read-once. Indeed, consider any minimal $S_{0} \subset\left\{x_{1}, \ldots, x_{n}\right\}$ such that $C\left(S_{0} \rightarrow 1\right)=1$ and $D\left(S_{0} \rightarrow 1\right)=0$. Such a set exists, since $C \rightarrow D$ is not a tautology. As $D\left(S_{0} \rightarrow 1\right)=0$, the set $S_{0}$ does not include any right set, and hence is a left minterm of $C \vee D$. As, $C\left(S_{0} \rightarrow 1\right)=1$, the set $S_{0}$ intersects both $C_{u}$ and $C_{v}$. Recall that $C_{u}, C_{v} \subset T$ and hence $\left|T \cap S_{0}\right| \geq 2$. By Theorem 1, $C \vee D$ does not compute a read-once function.

Otherwise (if there is no maxterm $T$ of $C \vee D$ that includes distinct clauses of $C$ ) we proceed to Step 3.

Step 3. For every clause $C_{u}$ and for every pair of distinct variables $p$ and $q$ from $C_{u}$ we check:

- whether there is a right minterm $S$ such that $\{p, q\} \subset S$ (this can be done in polynomial time since there are only polynomially many right minterms);

- whether there is a maxterm $T$ containing $C_{u}$.

The second check can be done in polynomial time using the following

Lemma 4. Assume that $C \rightarrow D$ is not a tautology and no maxterm of $C \vee D$ contains two distinct clauses of $C$. Then for any clause $C_{i}$ we can decide in polynomial-time whether there exists a maxterm $T$ of $C \vee D$ such that $C_{i} \subset T$.

If for some $C_{u}, p, q$ both questions answer in positive, then $C \vee D$ is not a read-once function. Indeed, in this case both the minterm $S$ and the maxterm $T$ include distinct variables $p$ and $q$, and by Theorem 1, $C \vee D$ does not compute a read-once function. Otherwise we proceed to Step 4 .

Step 4. For all $u \in\{1, \ldots, m\}$ and $v \in\{1, \ldots, l\}$ with $C_{u} \cap D_{v}=\varnothing$, and for all $p \in D_{v}$ and $q \in C_{u}$ we check:

- whether there is a left minterm $S$ such that $\{p, q\} \subset S$,

- whether there is a maxterm $T$ such that $C_{u} \cup\{p\} \subset T$.

Both checks can be performed in polynomial time by the following lemmas.

Lemma 5. There exists a polynomial-time algorithm which for any given pair of distinct variables $a, b \in\left\{x_{1}, \ldots, x_{n}\right\}$ decides, whether there is a left minterm $S$ of $C \vee D$ such that $\{a, b\} \subset S$.

Lemma 6. Assume that there is no maxterm of $C \vee D$ which contains two distinct clauses of $C$. Then for any given $C_{u}, D_{v}$ with $C_{u} \cap D_{v}=\varnothing$ and for any given $p \in D_{v}$ we can decide in polynomial time whether there exists a maxterm $T$ of $C$ such that $C_{u} \cup\{p\} \subset T$. 2

If for some $C_{u}, D_{v}, p, q$ both questions answer in positive, then $C \vee D$ does not compute a read-once function. Indeed, in this case both the maxterm $T$ and the minterm $S$ include distinct variables $p, q$ and by Theorem 1 . $C \vee D$ does not compute a read-once function.

\footnotetext{
${ }^{2}$ The proof of this lemma is almost identical to the proof of Lemma 4 Actually, it is possible to formulate a single lemma which implies both of them, but then the formulation of the lemma becomes immense.
} 
Otherwise the algorithm outputs the positive answer and halts. We have to show that in this case $C \vee D$ indeed computes a read-once function. For the sake of contradiction, assume that $C \vee D$ is not read-once. By Theorem 1 there is a maxterm $T$ of $C \vee D$ and a minterm $S$ of $C \vee D$ that have distinct common variables $p, q$. By Lemma $2 S$ is either a left or a right minterm. We will consider these two cases separately.

Case 1: $S$ is a right set, say $S=D_{j}$. Then $T$ contains some clause $C_{u}$ of $C$. We claim that $S \cap T \subset C_{u}$. Indeed, otherwise $S \cap T$ would include a variable $y \notin C_{u}$. Then $C(T \backslash\{y\} \rightarrow 0)=0$, as $C_{u} \subset T \backslash\{y\}$. And $D(T \backslash\{y\} \rightarrow 0)=0$, since $D$ is read-once and $T \cap D_{j}$ has at least 2 variables. We obtain contradiction, as $T$ is a maxterm of $C \vee D$.

Thus $T \cap S \subset C_{u}$. Hence there are two distinct variables $p$ and $q$ from $C_{u}$ such that $\{p, q\} \subset S$. Hence the algorithm must have halted on Step 3, a contradiction.

Case 2: $S$ is a left set. Again there is a clause $C_{u} \subset T$. Since $S$ is a left set, $S$ and $C_{u}$ have exactly one common variable $q$. By our assumption $T \cap S$ includes another variable $p \neq q$. Note that $p \notin C_{u}$ because otherwise $S \cap C_{u}$ has more than one variable.

Let $D_{v}$ be the (unique) right set that includes $p$ (here we use the assumption that every variable is in some right set). We claim that $D_{v}$ and $C_{u}$ are disjoint. For the sake of contradiction assume that there is a variable $y \in C_{u} \cap D_{v}$. Then $(C \vee D)(T \backslash\{p\} \rightarrow 0)=0$. Indeed, $C(T \backslash\{p\} \rightarrow 0)=0$, as $p \notin C_{u}$ and hence $C_{u} \subset T \backslash\{p\}$. And $D(T \backslash\{p\} \rightarrow 0)=0$, as $y \neq p$ (since $y$ is in $C_{u}$ and $p$ is not) and hence $T \backslash\{p\}$ still intersects $D_{v}$.

Thus $T$ is not a maxterm, and the contradiction shows that $D_{v}$ and $C_{u}$ are disjoint. Therefore, we have found $C_{u}, p, q, D_{v}$ such that $q \in C_{u}, p \in D_{v}$, $C_{u}$ and $D_{v}$ are disjoint and there is a left minterm $S$ and a maxterm $T$ with $\{p, q\} \subset S, C_{u} \cap\{p\} \subset T$. Hence the algorithm just have halted on Step 4, a contradiction. (End of Algorithm.)

\section{Lower bound}

We start this section with the proof of our variant of the Sausage Lemma (Theorem 3).

Proof (of Theorem (3). We reduce from a clique problem. Assume that we are given a simple undirected graph $G=(V, E)$ and an integer $k$ and we want to know whether there is a clique of size $k$ in $G$. The reduction is divided into two steps. On step 1 we introduce an auxiliary game between 3 players, Alice, Bob and Merlin. In this game Alice and Bob cooperatively play against Merlin. The rules of the game depend on $G, k$. The structure of this game resembles so-called GKS communication games (see [8]). We will show that there is a clique of size $k$ in $G$ if and only if Alice and Bob have a winning strategy in a game. On step 2 of the reduction we will construct (in polynomial time) a $\Lambda-\bigvee-\bigwedge$ depth-3 read-once monotone Boolean formula $\Psi$ with the following feature: Alice and Bob have a winning strategy in a game if and only if $\Psi \rightarrow D_{n}$ is not a tautology. The value of $n$ will depend on $k$ and on the size of $G$. 
The game. There is a room with a tape consisting of $k$ blank cells. The game has two phases. During the first phase Alice and Merlin are in the room and Bob is outside. He does not see what happens in a room. Alice and Merlin interact according to the following protocol. At the beginning Merlin points out to one of $k$ cells. Then Alice writes some vertex $u$ of $G$ in this cell. Finally, Merlin writes some vertex $v$ of $G$ in some other cell. The only restriction is that $v$ should not be connected with $u$ by an edge of $G$ (for example, $v$ can be equal to $u$, we assume that there are no self-loops in $G$ ). The first phase is finished.

Then, during the second phases, Bob comes in and Alice with Merlin are outside. Bob sees that exactly two cells of a tape are not blank - each of these two cells contains a vertex of a graph. To win, Bob should determine which cell was touched first. More precisely, the cell which was touched first is the one to which Merlin pointed out in the beginning and in which Alice wrote her vertex.

More formally, let CONF denote the following set:

$$
\mathrm{CONF}=\{\{(i, u),(j, v)\}: i, j \in\{1, \ldots, k\}, u, v \in V, i \neq j,\{u, v\} \notin E\},
$$

Note that CONF encodes all possible configurations Bob can see when he comes in.

A strategy of Alice in this game is a function $S_{A}:\{1,2, \ldots, k\} \rightarrow V$, a strategy of Bob is a function $S_{B}: \mathrm{CONF} \rightarrow\{1,2, \ldots, k\}$ and a strategy of Merlin is a triple $\left(i, S_{M}^{1}, S_{M}^{2}\right)$, where $i \in\{1,2, \ldots, k\}$ and

$$
S_{M}^{1}: V \rightarrow\{1,2, \ldots, k\}, \quad S_{M}^{2}: V \rightarrow V .
$$

This triple should satisfy the following condition: for every $u \in V$ it holds that $S_{M}^{1}(u) \neq i$ and $\left\{u, S_{M}^{2}(u)\right\} \notin E$. We call a pair $\left(S_{A}, S_{B}\right)$ a winning strategy, if for every Merlin's strategy $\left(i, S_{M}^{1}, S_{M}^{2}\right)$ it holds that

$$
S_{B}\left(\left\{\left(i, S_{A}(i)\right),\left(S_{M}^{1}\left(S_{A}(i)\right), S_{M}^{2}\left(S_{A}(i)\right)\right)\right\}\right)=i .
$$

Let us show that if there is clique of size $k$ in $G$, then Alice and Bob have a winning strategy in the above game. Indeed, assume that $w_{1}, \ldots, w_{k} \in V$ form a $k$-clique in $G$. Alice's strategy is the following: if Merlin points out to the $i^{t h}$ cell, then she writes $w_{i}$ in it. Assume that after that Merlin picks $j^{\text {th }}$ cell and writes something in it. How can Bob distinguish the $i^{\text {th }}$ cell from the $j^{\text {th }}$ cell? The point is that for $v$ which is written by Merlin in the $j^{\text {th }}$ cell we have that $v \neq w_{j}$, because $w_{i}$ and $w_{j}$ are connected by an edge. This allows Bob to win.

On the other hand, assume that Alice and Bob have a winning strategy in a game. Let $v_{i}$ be the vertex which Alice writes according to this strategy when Merlin points out to the $i^{\text {th }}$ cell. Let us show that $v_{1}, \ldots, v_{k}$ form a clique in $G$. For contradiction assume that there is $i \neq j$ such that $v_{i}$ and $v_{j}$ are not connected by edge. Note that there are two strategies of Merlin resulting into the same (from the Bob's point of view) configuration, namely:

- Merlin points to the $i^{\text {th }}$ cell and then writes $v_{j}$ in the $j^{\text {th }}$ cell;

- Merlin points to the $j^{\text {th }}$ cell and then writes $v_{i}$ in the $i^{\text {th }}$ cell. 
Both these strategies are legal since $\left\{v_{i}, v_{j}\right\} \notin E$. This contradicts the fact that from this configuration Bob can determine the cell which was touched first.

Now let us proceed to the second step of a reduction. There are exactly $2 \cdot|\mathrm{CONF}|$ outcomes of Alice-Merlin interaction (for each $c \in$ CONF there are two outcomes resulting in $c$ ). Introduce a Boolean variable for each possible outcome. Namely, let $x_{j, v}^{i, u}$ correspond to an outcome in which Merlin picked the $i^{t h}$ cell, Alice wrote $u$ in the $i^{\text {th }}$ cell and then Merlin wrote $v$ in the $j^{\text {th }}$ cell.

Every assignment to these variables, on which the value of $D_{n}$ equals 0 , determines Bob's strategy. Here $D_{n}=\bigvee_{\{(i, u),(j, v)\} \in \mathrm{CONF}} x_{j, v}^{i, u} \wedge x_{i, u}^{j, v}$. Namely, for $c=\{(i, u),(j, v)\}$ define:

$$
S_{B}(c)= \begin{cases}i & \text { if } x_{j, v}^{i, u}=1 \\ j & \text { if } x_{i, u}^{j, v}=1\end{cases}
$$

For technical reasons, if both $x_{j, v}^{i, u}$ and $x_{i, u}^{j, v}$ are set to 0 , define $S_{B}(c)$ somehow in such a way that $S_{B}(c) \neq i, j$. Note that it never happens that both $x_{j, v}^{i, u}$ and $x_{i, u}^{j, v}$ are set to 1 (otherwise $S_{B}(c)$ would be undetermined for $c=\{(i, u),(j, v)\}$ ). It is also clear that every Bob's strategy can be encoded in this way by some assignment of variables on which $D_{n}=0$.

Once a Bob's strategy (or equivalently, an assignment to $x_{j, v}^{i, u}$ ) is fixed, the game is just between Alice and Merlin. Alice wins if and only if an outcome of her interaction with Merlin corresponds to a variable which is set to 1. Indeed, if an outcome corresponds to $x_{j, v}^{i, u}$, then Bob should output $i$ and it happens if and only if $x_{j, v}^{i, u}$ is set to 1 (here in $\left(\Longrightarrow\right.$ )-direction we use the fact that if both $x_{j, v}^{i, u}$ and $x_{i, u}^{j, v}$ are set to 0 , then $\left.S_{B}(c) \neq i, j\right)$. This means that Alice has a winning strategy if and only if $\Psi=1$, where

$$
\Psi=\bigwedge_{i=1}^{k} \bigvee_{u \in V} \bigwedge_{\substack{(j, v): \\\{(i, u),(j, v)\} \in \mathrm{CONF}}} x_{j, v}^{i, u}
$$

( $\bigwedge$-gates correspond to Merlin's moves and $\bigvee$-gate corresponds to Alice's move). In other words, Alice and Bob have a winning strategy if and only if there is an assignment to $x_{j, v}^{i, u}$ such that $D_{n}$ is false and $\Psi$ is true or, equivalently, $\Psi \rightarrow D_{n}$ is not a tautology.

To derive Corollary 1 we need the following simple Lemma.

Lemma 7. A function

$$
f\left(w_{1}, w_{2}, w_{3}, w_{4}\right)=w_{2} w_{3} w_{4} \vee w_{1} w_{3} w_{4} \vee w_{1} w_{2} w_{4} \vee w_{1} w_{2} w_{3}
$$

is not read-once.

Proof. Note that $\left\{w_{1}, w_{2}, w_{3}\right\}$ is a minterm of $f$ and $\left\{w_{1}, w_{2}\right\}$ is a maxterm of $f$. Hence by Theorem $1 f$ is not read-once. 
Proof (of Corollary [1). Assume that $\Psi$ is a $\Lambda-\bigvee-\bigwedge$ depth-3 read-once monotone Boolean formula over $x_{1}, y_{1}, \ldots, x_{n}, y_{n}$ and $D_{n}=\bigvee_{i=1}^{n} x_{i} y_{i}$. It is enough to show that $\Psi \rightarrow D_{n}$ is a tautology if and only if

$$
\left(\Psi \wedge\left(w_{1} w_{3} \vee w_{2} w_{4}\right)\right) \wedge\left(D_{n} \vee w_{1} w_{2} \vee w_{3} w_{4}\right)
$$

computes a read-once function. Indeed, if $\Psi \rightarrow D_{n}$ is a tautology, then

$$
\left(\Psi \wedge\left(w_{1} w_{3} \vee w_{2} w_{4}\right)\right) \rightarrow\left(D_{n} \vee w_{1} w_{2} \vee w_{3} w_{4}\right)
$$

is also a tautology. Hence

$$
\left(\Psi \wedge\left(w_{1} w_{3} \vee w_{2} w_{4}\right)\right) \wedge\left(D_{n} \vee w_{1} w_{2} \vee w_{3} w_{4}\right)=\Psi \wedge\left(w_{1} w_{3} \vee w_{2} w_{4}\right)
$$

and the latter is a read-once formula. On the other hand assume for contradiction that $\Psi \rightarrow D_{n}$ is not a tautology, but there is a read-once formula $\Phi\left(x_{1}, y_{1}, \ldots, x_{n}, y_{n}, w_{1}, \ldots, w_{4}\right)$, which is logically equivalent to

$$
\left(\Psi \wedge\left(w_{1} w_{3} \vee w_{2} w_{4}\right)\right) \wedge\left(D_{n} \vee w_{1} w_{2} \vee w_{3} w_{4}\right) .
$$

Let $a_{1}, b_{1}, \ldots, a_{n}, b_{n}$ be an assignment to $x_{1}, y_{1}, \ldots, x_{n}, y_{n}$ such that

$$
\Psi\left(a_{1}, b_{1} \ldots, a_{n}, b_{n}\right)=1, \quad D_{n}\left(a_{1}, b_{1} \ldots, a_{n}, b_{n}\right)=0 .
$$

Substitute $x_{1} \rightarrow a_{1}, y_{1} \rightarrow b_{1}, \ldots x_{n} \rightarrow a_{n}, y_{n} \rightarrow b_{n}$ in $\Phi$. The resulting read-once formula will be

$$
\begin{aligned}
\Phi\left(a_{1}, b_{1}, \ldots, a_{n}, b_{n}, w_{1}, \ldots, w_{4}\right) & =\left(w_{1} w_{3} \vee w_{2} w_{4}\right) \wedge\left(w_{1} w_{2} \vee w_{3} w_{4}\right) \\
& =w_{2} w_{3} w_{4} \vee w_{1} w_{3} w_{4} \vee w_{1} w_{2} w_{4} \vee w_{1} w_{2} w_{3} .
\end{aligned}
$$

Thus we obtain a read-once formula for $w_{2} w_{3} w_{4} \vee w_{1} w_{3} w_{4} \vee w_{1} w_{2} w_{4} \vee w_{1} w_{2} w_{3}$, this a contradiction with Lemma 7.

Acknowledgments The author would like to thank Alexander Shen and Nikolay Vereshchagin for help in writing this paper. The author would like to thank Vladimir Gurvich for pointing out to [7].

\section{References}

1. Elbassioni, K., Makino, K., and Rauf, I. On the readability of monotone boolean formulae. Journal of combinatorial optimization 22, 3 (2011), 293-304.

2. Gurvich, V. It is a conp-complete problem to decide whether a positive $\vee-\wedge$ formula of depth 3 defines a read-once or respectively quadratic boolean function. Rutcor Research Report (2010).

3. Golumbic, M. C., And Gurvich, V. Read-once functions. Boolean Functions: Theory, Algorithms and Applications (2009).

4. Golumbic, M. C., Mintz, A., And Rotics, U. An improvement on the complexity of factoring read-once boolean functions. Discrete Applied Mathematics 156, 10 (2008), 1633-1636. 
5. Gurvich, V. A. Repetition-free boolean functions. Uspekhi Matematicheskikh Nauk 32, 1 (1977), 183-184.

6. Büning, H. And Lettmann, T. Propositional logic: deduction and algorithms Cambridge University Press, 48 (1999)

7. Boros, E., Elbassioni, K., Gurvich, V., and Makino, K. Generating Vertices of Polyhedra and Related Problems of Monotone Generation. CRM Proceedings and Lecture Notes, 48 (2009)

8. Gilmer, J., Kouckỳ, M., And SAKs, M. A new approach to the sensitivity conjecture. Proceedings of the 2015 Conference on Innovations in Theoretical Computer Science, (2015), 247-254.

\section{Appendix}

\section{A Proof of Lemma 3}

Let $T$ be a maxterm of $C \vee D$ and $C_{u}, C_{v} \subset T$. For the sake of contradiction assume that there is $j \in\{1, \ldots, l\}$ such that $\left|\left(C_{u} \cup C_{v}\right) \cap D_{j}\right| \geq 2$. Pick any two distinct $p, q \in\left(C_{u} \cup C_{v}\right) \cap D_{j}$. Let us show that $(C \vee D)(T \backslash\{q\} \rightarrow 0)=0$. To show that $C(T \backslash\{q\} \rightarrow 0)=0$ observe that $C_{u}$ or $C_{v}$ does not contain $q$ and hence $C_{u} \subset T \backslash\{q\}$ or $C_{v} \subset T \backslash\{q\}$. To show that $D(T \backslash\{q\} \rightarrow 0)=0$ observe that $D(T \rightarrow 0)=0$ and hence $T$ intersects all sets $D_{1}, \ldots, D_{l}$. Since $q \in D_{j}$ and hence $q \notin D_{i}$ for all $i \neq j$, the set $T \backslash\{q\}$ still intersects $D_{i}$ for all $i \neq j$. And it intersects $D_{j}$ since $p \in C_{u} \cup C_{v} \subset T$ and $p$ was not removed from $T$. Since $T$ is a maxterm, this is a contradiction.

On the other hand, assume that for every $j \in\{1, \ldots, l\}$ it holds that $\mid\left(C_{u} \cup\right.$ $\left.C_{v}\right) \cap D_{j} \mid \leq 1$. We have to find a maxterm $T$ that includes both $C_{u}$ and $C_{v}$. Start with $T=C_{u} \cup C_{v}$. Then for all $j$ such that $D_{j}$ does not intersect $C_{u} \cup C_{v}$ pick a variable from $D_{j}$ and include it in $T$. In this way we make $T$ intersect every $D_{j}$ in exactly one point. In particular, $D(T \rightarrow 0)=0$ and $C(T \rightarrow 0)=0$. On the other hand, every proper subset $T^{\prime}$ of $T$ is disjoint with at least one $D_{j}$ and hence $D\left(T^{\prime} \rightarrow 0\right)=1$. This shows that $T$ is a maxterm.

\section{B Proof of Lemma 4}

Since $C \rightarrow D$ is not a tautology, $C_{i}$ is non-empty and intersects with some $D_{j}$. Further, without loss of generality we may assume that:

$-i=1$;

$-C_{1}$ intersects with $D_{1}, \ldots D_{r}$ and $C_{1}$ is disjoint with $D_{r+1}, \ldots D_{l}$ for some $1 \leq r \leq l$;

$-C_{2}, \ldots, C_{s}$ all intersect with $D_{1} \cup D_{2} \cup \ldots \cup D_{r}$ and $C_{s+1}, \ldots, C_{m}$ are all disjoint with $D_{1} \cup D_{2} \cup \ldots \cup D_{r}$ for some $1 \leq s \leq m$.

From the fact that $D_{1} \cup D_{2} \cup \ldots \cup D_{l}=\left\{x_{1}, \ldots, x_{n}\right\}$ we may derive that:

$$
C_{s+1}, \ldots, C_{m} \subset D_{r+1} \cup \ldots \cup D_{l}
$$


Define an auxiliary CNF $\widehat{C}=C_{s+1} \wedge \ldots \wedge C_{m}$ and an auxiliary DNF $\widehat{D}=$ $D_{r+1} \vee \ldots \vee D_{l}$. Note that $\widehat{C}$ and $\widehat{D}$ are over variables from $D_{r+1} \cup \ldots \cup D_{l}$ (this follows from (11)).

We claim that there exists $T$ such that $T$ is a maxterm of $C \vee D$ and $C_{1} \subset T$ if and only if $\widehat{C} \rightarrow \widehat{D}$ is not a tautology (the latter by Lemma 1 can be verified in polynomial time).

$(\Leftarrow)$. Assume that $\widehat{C} \rightarrow \widehat{D}$ is not a tautology. Then there exists $\widehat{T} \subset D_{r+1} \cup$ $\ldots \cup D_{l}$ such that

$$
\begin{gathered}
C_{s+1} \not \subset \widehat{T}, \ldots, C_{m} \not \subset \widehat{T} ; \\
\left|D_{r+1} \cap \widehat{T}\right|=1, \ldots,\left|D_{l} \cap \widehat{T}\right|=1 ;
\end{gathered}
$$

(take minimal $\widehat{T} \subset D_{r+1} \cup \ldots \cup D_{l}$ such that $\widehat{C}(\widehat{T} \rightarrow 0)=1$ and $\widehat{D}(\widehat{T} \rightarrow 0)=0$ ).

Let us show that $T=\widehat{T} \cup C_{1}$ is the maxterm of $C \vee D$. First of all, let us verify that $(C \vee D)(T \rightarrow 0)=0$. Indeed,

- $C(T \rightarrow 0)=0$ because $C_{1} \subset T$;

- $D(T \rightarrow 0)=0$ because every $D_{1}, \ldots, D_{l}$ intersects with $T$; namely, $D_{1}, \ldots D_{r}$ intersect $C_{1}$ and $D_{r+1}, \ldots, D_{l}$ intersect $\widehat{T}$.

Now, assume that $T^{\prime} \subset T$ and $(C \vee D)\left(T^{\prime} \rightarrow 0\right)=0$. Let us show that this is possible only when $T^{\prime}=T$.

Since $D\left(T^{\prime} \rightarrow 0\right)=0$, we have that $T^{\prime}$ intersects with every $D_{1}, \ldots, D_{l}$. From the fact that $C_{1}$ is disjoint with $D_{r+1}, \ldots, D_{l}$ and from (3) it follows that $\widehat{T} \subset T^{\prime}$.

It remains to show that $C_{1} \subset T^{\prime}$. This follows from the assumption that $C\left(T^{\prime} \rightarrow 0\right)=0$. Indeed, then at least one clause of $C$ should be the subset of $T^{\prime}$. Assume that this clause is $C_{u}$. If $C_{u} \neq C_{1}$, then $C_{u} \subset \widehat{T}$. There are two cases:

- The first case. Assume that $C_{u} \in\left\{C_{2}, \ldots, C_{s}\right\}$. Then $C_{u} \subset \widehat{T} \subset D_{r+1} \cup \ldots \cup$ $D_{l}$ intersects with $D_{1} \cup D_{2} \cup \ldots \cup D_{r}$, but the latter is impossible.

- The second case. Assume that $C_{u} \in\left\{C_{s+1}, \ldots, C_{m}\right\}$. This case contradicts (2).

$(\Rightarrow)$ Assume that $T$ is the maxterm of $C \vee D$ such that $C_{1} \subset T$. Define $\widehat{T}=T \backslash C_{1}$. Later we will show that $\widehat{C}(\widehat{T} \rightarrow 0)=1, \widehat{D}(\widehat{T} \rightarrow 0)=0$ and hence $\widehat{C} \rightarrow \widehat{D}$ is not a tautology. But at first we should verify that $\widehat{T} \subset D_{r+1} \cup \ldots \cup D_{l}$ (recall that $\widehat{C}, \widehat{D}$ are over variables from $D_{r+1} \cup \ldots \cup D_{l}$ ).

To show that $\widehat{T} \subset D_{r+1} \cup \ldots \cup D_{l}$ assume for contradiction that $\widehat{T}$ intersects $D_{1} \cup D_{2} \cup \ldots \cup D_{r}$ and let $q$ be the variable which lies in their intersection. Note that $q \notin C_{1}$ (this is because $q \in \widehat{T}=T \backslash C_{1}$ ). Let us demonstrate that for such $q$ we have that $(C \vee D)(T \backslash\{q\} \rightarrow 0)=0$ (this is already a contradiction since $T$ is a maxterm). Indeed, $C(T \backslash\{q\} \rightarrow 0)=0$ since $C_{1} \subset T \backslash\{q\}$. Further, we should show that $T \backslash\{q\}$ intersects every $D_{1}, \ldots D_{l}$ and hence $D(T \backslash\{q\} \rightarrow 0)=0$. Indeed:

- $T \backslash\{q\}$ intersects $D_{1}, \ldots, D_{r}$ because of $C_{1}$; 
- $T \backslash\{q\}$ intersects $D_{r+1}, \ldots, D_{l}$ because of the following two reasons: (a) $D(T \rightarrow 0)=0$ and hence $T$ intersects every $D_{r+1}, \ldots, D_{l}$; (b) $q$ is not in $D_{r+1} \cup \ldots \cup D_{l}$.

Thus it remains to show that $\widehat{C}(\widehat{T} \rightarrow 0)=1$ and $\widehat{D}(\widehat{T} \rightarrow 0)=0$. To show that the first equality is true assume for contradiction that there is $C_{u} \in$ $\left\{C_{s+1}, \ldots, C_{m}\right\}$ such that $C_{u} \subset \widehat{T}=T \backslash C_{1}$. But then $C_{u} \subset T$. This contradicts the assumption that there is no maxterm of $C \vee D$ which contains two distinct clauses of $C$.

To show that the second equality $(\widehat{D}(\widehat{T} \rightarrow 0)=0)$ is true, observe that $\widehat{T}$ intersects every $D_{r+1}, \ldots, D_{l}$. This is true because $D(\widehat{T} \rightarrow 0)=0$ and hence $T$ intersects $D_{r+1}, \ldots, D_{l}$; but $C_{1}$ by assumption is disjoint with $D_{r+1}, \ldots, D_{l}$ and hence $T \backslash C_{1}$ still intersects them.

\section{Proof of Lemma 5}

If there is $C_{i}$ such that $a, b \in C_{i}$ or $\{a, b\} \not \subset C_{1} \cup \ldots \cup C_{m}$, then no left minterm $S$ can contain both $a$ and $b$. From now we assume that this is not the case, i.e. there is no $C_{i}$ which contains both $a$ and $b$ and $a, b \in C_{1} \cup \ldots \cup C_{m}$. Let $\widehat{C} \vee \widehat{D}$ be obtained from $C \vee D$ by setting $a, b$ to 1 . In other words, $\widehat{C}$ is obtained from $C$ by erasing all clauses containing $a$ or $b$ and $\widehat{D}$ is obtained from $D$ by erasing $a$ and $b$. Assume without loss of generality that $C_{1}, C_{2}$ are erased clauses, $a \in C_{1}, b \in C_{2}$ and $D_{1}, \ldots, D_{r}$ are conjunctions containing $a$ or $b$ (note that $r$ is either 1 or 2). Then $\widehat{C}$ and $\widehat{D}$ can be written as

$$
\begin{gathered}
\widehat{C}=C_{3} \wedge \ldots \wedge C_{m}, \\
\widehat{D}=\left(D_{1} \backslash\{a, b\}\right) \vee \ldots \vee\left(D_{r} \backslash\{a, b\}\right) \vee D_{r+1} \vee \ldots \vee D_{l} .
\end{gathered}
$$

We assert that there is a left minterm $S$ containing $a$ and $b$ iff $\widehat{C} \rightarrow \widehat{D}$ is not a tautology. The latter by Lemma 1 can be verified in polynomial times.

$(\Leftarrow)$ Assume that $\widehat{C} \rightarrow \widehat{D}$ is not a tautology. Take minimal $\widehat{S} \subset\left\{x_{1}, \ldots, x_{n}\right\} \backslash$ $\{a, b\}$ such that $\widehat{C}(\widehat{S} \rightarrow 1)=1, \widehat{D}(\widehat{S} \rightarrow 1)=0$. Obviously, such $\widehat{S}$ satisfies the following two conditions:

$$
\begin{gathered}
\widehat{S} \subset C_{3} \cup \ldots \cup C_{m}, \quad\left|\widehat{S} \cap C_{3}\right|=1, \ldots,\left|\widehat{S} \cap C_{m}\right|=1, \\
D_{1} \backslash\{a, b\} \not \subset \widehat{S}, \ldots, D_{r} \backslash\{a, b\} \not \subset \widehat{S}, D_{r+1} \not \subset \widehat{S}, \ldots, D_{l} \not \subset \widehat{S} .
\end{gathered}
$$

Now, define $S=\widehat{S} \cup\{a, b\}$. Let us show that $S$ is a left minterm of $C \vee D$. From (5) it follows that there is no $j \in\{1, \ldots, l\}$ such that $D_{j} \subset S$. Hence $S$ contains no right set as a proper subset. Thus it remains to show by Lemma 2 that $S$ is a left set. Since $a, b$ are from $C_{1} \cup \ldots \cup C_{m}$, we have that

$$
S \subset\left(\{a, b\} \cup C_{3} \cup \ldots \cup C_{m}\right) \subset C_{1} \cup \ldots \cup C_{m} .
$$


Moreover, $S$ intersects every clause of $C$ in exactly one point. For $C_{3}, \ldots, C_{m}$ this follows from (41) and from the fact that $C_{3}, \ldots, C_{m}$ contain neither $a$ nor $b$. For $C_{1}, C_{2}$ this is true because: (a) $\widehat{S}$ is disjoint with $C_{1}, C_{2}$; (b) $a \in C_{1}, b \in C_{2}$.

$(\Rightarrow)$ Assume that there is a left minterm $S$ of $C \vee D$ containing $a$ and $b$. Define $\widehat{S}=S \backslash\{a, b\}$. Let us show that $\widehat{S}$ intersects every $C_{3}, \ldots C_{m}$. Indeed, this is true for $S$ and $a, b$ are not from $C_{3} \cup \ldots \cup C_{m}$. Hence $\widehat{C}(\widehat{S} \rightarrow 1)=1$. On the other hand, $\widehat{D}(\widehat{S} \rightarrow 1)=0$, since:

- $D_{1} \backslash\{a, b\} \not \subset \widehat{S}, \ldots D_{r} \backslash\{a, b\} \not \subset \widehat{S}$ because otherwise at least one $D_{1}, \ldots, D_{r}$ is the subset of $S=\widehat{S} \cup\{a, b\}$;

- $D_{r+1} \not \subset \widehat{S}, \ldots, D_{l} \not \subset \widehat{S}$ because it is true even for $S$.

Thus $\widehat{C} \rightarrow \widehat{D}$ is not a tautology.

\section{Proof of Lemma 6}

Without loss of generality we may assume that:

$-i=j=1$;

- $C_{1}$ intersects with $D_{2}, \ldots D_{r}$ and $C_{1}$ is disjoint with $D_{r+1}, \ldots D_{l}$ for some $1 \leq r \leq l$;

$-C_{2}, \ldots, C_{s}$ all intersect with $D_{1} \backslash\{p\} \cup D_{2} \cup \ldots \cup D_{r}$ and $C_{s+1}, \ldots, C_{m}$ are all disjoint with $D_{1} \backslash\{p\} \cup D_{2} \cup \ldots \cup D_{r}$ for some $1 \leq s \leq m$.

From the fact that $D_{1} \cup D_{2} \cup \ldots \cup D_{l}=\left\{x_{1}, \ldots, x_{n}\right\}$ we may derive that:

$$
C_{s+1}, \ldots, C_{m} \subset\{p\} \cup D_{r+1} \cup \ldots \cup D_{l} .
$$

Now, define:

$$
\widehat{C}_{s+1}=C_{s+1} \backslash\{p\}, \ldots, \widehat{C}_{m}=C_{m} \backslash\{p\} .
$$

Further, define an auxiliary CNF $\widehat{C}=\widehat{C}_{s+1} \wedge \ldots \wedge \widehat{C}_{m}$ and an auxiliary DNF $\widehat{D}=D_{r+1} \vee \ldots \vee D_{l}$. Note that $\widehat{C}$ and $\widehat{D}$ are over variables from $D_{r+1} \cup \ldots \cup D_{l}$ (this follows from (6)).

We claim that there exists $T$ such that $T$ is a maxterm of $C \vee D$ and $C_{1} \cup\{p\} \subset$ $T$ if and only if $\widehat{C} \rightarrow \widehat{D}$ is not a tautology (the latter by Lemma 1 can be verified in polynomial time).

$(\Leftarrow)$. Assume that $\widehat{C} \rightarrow \widehat{D}$ is not a tautology. Then there exists $\widehat{T} \subset D_{r+1} \cup$ $\ldots \cup D_{l}$ such that

$$
\begin{gathered}
\widehat{C}_{s+1} \not \subset \widehat{T}, \ldots, \widehat{C}_{m} \not \subset \widehat{T} ; \\
\left|D_{r+1} \cap \widehat{T}\right|=1, \ldots,\left|D_{l} \cap \widehat{T}\right|=1 ;
\end{gathered}
$$

(take minimal $\widehat{T} \subset D_{r+1} \cup \ldots \cup D_{l}$ such that $\widehat{C}(\widehat{T} \rightarrow 0)=1$ and $\left.\widehat{D}(\widehat{T} \rightarrow 0)=0\right)$.

Let us show that $T=\widehat{T} \cup C_{1} \cup\{p\}$ is a maxterm of $C \vee D$. First of all, let us verify that $(C \vee D)(T \rightarrow 0)=0$. Indeed, 
- $C(T \rightarrow 0)=0$ because $C_{1} \subset T$;

- $D(T \rightarrow 0)=0$ because every $D_{1}, \ldots, D_{l}$ intersects with $T$; namely, $D_{1}$ contains $p, D_{2}, \ldots D_{r}$ intersect $C_{1}$ and $D_{r+1}, \ldots, D_{l}$ intersect $\widehat{T}$.

Now, assume that $T^{\prime} \subset T$ and $(C \vee D)\left(T^{\prime} \rightarrow 0\right)=0$. Let us show that this is possible only when $T^{\prime}=T$.

Since $D\left(T^{\prime} \rightarrow 0\right)=0$, we have that $T^{\prime}$ intersects with every $D_{1}, \ldots, D_{l}$. From the fact that $C_{1} \cup\{p\}$ is disjoint with $D_{r+1}, \ldots, D_{l}$ and from (8) it follows that $\widehat{T} \subset T^{\prime}$. Since $C_{1}$ and $\widehat{T}$ are disjoint with $D_{1}$ and $T^{\prime}$ intersects $D_{1}$, we have that $p \in T^{\prime}$.

It remains to show that $C_{1} \subset T^{\prime}$. This follows from the assumption that $C\left(T^{\prime} \rightarrow 0\right)=0$. Indeed, then at least one clause of $C$ should be the subset of $T^{\prime}$. Assume that this clause is $C_{u}$. If $C_{u} \neq C_{1}$, then $C_{u} \subset \widehat{T} \cup\{p\}$. There are two cases:

- The first case. Assume that $C_{u} \in\left\{C_{2}, \ldots, C_{s}\right\}$. Then $C_{u} \subset \widehat{T} \cup\{p\} \subset$ $\{p\} \cup D_{r+1} \cup \ldots \cup D_{l}$ intersects with $D_{1} \backslash\{p\} \cup D_{2} \cup \ldots \cup D_{r}$, but the latter is impossible.

- The second case. Assume that $C_{u} \in\left\{C_{s+1}, \ldots, C_{m}\right\}$. Then $\widehat{C}_{u}=C_{u} \backslash\{p\} \subset$ $\widehat{T}$, and the latter contradicts (7).

$(\Rightarrow)$ Assume that $T$ is the maxterm of $C \vee D$ such that $C_{1} \cup\{p\} \subset T$. Define $\widehat{T}=T \backslash\left(C_{1} \cup\{p\}\right)$. Later we will show that $\widehat{C}(\widehat{T} \rightarrow 0)=1, \widehat{D}(\widehat{T} \rightarrow 0)=0$ and hence $\widehat{C} \rightarrow \widehat{D}$ is not a tautology. But at first we should verify that $\widehat{T} \subset$ $D_{r+1} \cup \ldots \cup D_{l}$ (recall that $\widehat{C}, \widehat{D}$ are over variables from $D_{r+1} \cup \ldots \cup D_{l}$ ).

To show that $\widehat{T} \subset D_{r+1} \cup \ldots \cup D_{l}$ assume for contradiction that $\widehat{T}$ intersects $D_{1} \cup D_{2} \cup \ldots \cup D_{r}$ and let $q$ be the variable which lies in their intersection. Note that $q \neq p$ and $q \notin C_{1}$ (this is because $\left.q \in \widehat{T}=T \backslash\left(C_{1} \cup\{p\}\right)\right)$. Let us demonstrate that for such $q$ we have that $(C \vee D)(T \backslash\{q\} \rightarrow 0)=0$ (this gives us a contradiction since $T$ is a maxterm). Indeed, $C(T \backslash\{q\} \rightarrow 0)=0$ since $C_{1} \subset T \backslash\{q\}$. Further, we should show that $T \backslash\{q\}$ intersects every $D_{1}, \ldots D_{l}$ and hence $D(T \backslash\{q\} \rightarrow 0)=0$. Indeed:

- $T \backslash\{q\}$ intersects $D_{1}$ because of $p$;

- $T \backslash\{q\}$ intersects $D_{2}, \ldots, D_{r}$ because of $C_{1}$;

- $T \backslash\{q\}$ intersects $D_{r+1}, \ldots, D_{l}$ because of the following two reasons: (a) $D(T \rightarrow 0)=0$ and hence $T$ intersects every $D_{r+1}, \ldots, D_{l}$; (b) $q$ is not in $D_{r+1}, \ldots, D_{l}$.

Thus it remains to show that $\widehat{C}(\widehat{T} \rightarrow 0)=1$ and $\widehat{D}(\widehat{T} \rightarrow 0)=0$. To show that the first equality is true assume for contradiction that there is $\widehat{C}_{u} \in$ $\left\{\widehat{C}_{s+1}, \ldots, \widehat{C}_{m}\right\}$ such that $\widehat{C}_{u} \subset \widehat{T}=T \backslash\left(C_{1} \cup\{p\}\right)$. But $\widehat{C}_{u}=C_{u} \backslash\{p\}$ and hence $C_{u} \subset T$. This contradicts the assumption that there is no maxterm of $C \vee D$ which contains two distinct clauses of $C$.

To show that the second equality $(\widehat{D}(\widehat{T} \rightarrow 0)=0)$ is true, observe that $\widehat{T}$ intersects every $D_{r+1}, \ldots, D_{l}$. This follows from the following two observations: (a) $D(T \rightarrow 0)=0$ and hence $T$ intersects every $D_{r+1}, \ldots D_{l}$, (b) $\{p\} \cup C_{1}$ is disjoint with $D_{r+1}, \ldots, D_{l}$. 